\title{
Impact of Time of Sowing, Spacing and Seed Rate on Potential Seed Production and Fodder Quality of Cowpea /Vigna Unguiculata (L.) Walp
}

\author{
Dr. Rima Taipodia ${ }^{1}$ and A. T. Nabam ${ }^{2}$ \\ STO O/O Deputy Commissioner, Dibang Valley, Annini, Arunachal Pradesh \\ Fodder Development Officer, Directorate of Animal Husb. \& Vety. Govt. of Arunachal Pradesh, \\ Nirjuli
}

\begin{abstract}
To study the Impact Of Time Of Sowing, Spacing And Seed Rate On Potential Seed Production And Fodder Quality Of Cowpea, the field trial was conducted in kharif 2011 on medium deep black clay soil under rainfed condition at Directorate of Animal Husb. \& Vety. Govt. of Arunachal Pradesh, Nirjuli. The treatments comprised combination of three times of sowing (June second fortnight, July first fortnight and July second fortnight), two-row spacing (30 cm and 45 $\mathrm{cm})$ and three seed rates $\left(20,25\right.$ and $\left.30 \mathrm{~kg} \mathrm{ha}^{-1}\right)$. With three replications, in split-split plot design, the trial was laid out. Compared to July first fortnight and July second fortnight sowing, significantly higher seed yield, haulm yield and harvest index $\left(923 \mathrm{~kg} \mathrm{ha}^{-1}, 4440 \mathrm{~kg} \mathrm{ha}^{-1}\right.$ and 0.30 respectively) were recorded in cowpea sown in June second fortnight. Significantly, higher growth parameters at harvest viz., leaf area index (2.19), fresh weight (370.22 $\mathrm{g}$ plant $\left.{ }^{-1}\right)$, total dry matter $\left(63.32 \mathrm{~g} \mathrm{plant}^{-1}\right)$ and dry matter accumulation in leaves, stem and reproductive parts were also recorded in June second fortnight sowing. Time of sowing did not significantly influence the proximate quality parameters of seed and haulm and the seed quality parameters. Compared to 45 $\mathrm{cm}$, significantly higher growth and yield attributes, seed yield (741 $\left.\mathrm{kg} \mathrm{ha}^{-1}\right)$ and haulm yield $(4196$ $\mathrm{kg} \mathrm{ha}^{-1}$ ) was recorded in row spacing of $30 \mathrm{~cm}$. The proximate quality of seed and haulm and also seed quality were not influenced significantly by the row spacing. Growth, yield, proximate quality of seed and haulm and seed quality of cowpea were not significantly influenced by the Seed rate. in fodder cowpea with June second fortnight sowing with $30 \mathrm{~cm}$ row spacing at $30 \mathrm{~kg} \mathrm{ha}^{-1}$ seed rate for seed yield (1054 $\left.\mathrm{kg} \mathrm{ha}^{-1}\right)$ and haulm yield (4968 $\left.\mathrm{kg} \mathrm{ha}^{-1}\right)$, significantly higher interaction was recorded.
\end{abstract}

Key Words: fodder; yield; growth parameters, quality parameters.

\section{Introduction}

India is house to $15 \%$ (540 million) world cattle population (Singh, 2003) and 16\% of human population to be sustained and progressed on $2 \%$ of total geographical areas.

Throughout the last decade, the dairy industry in the country has exposed impressive growth and now India is the largest producer of milk. Singh, (2003) reported that current availability of green/dry fodder is estimated at 390 and 443 million tones, respectively. Owing to feed/fodder shortages which lean to be even more severe during natural calamities, efforts to boost livestock productivity are constrained. In view of the precedence for food grains, oilseeds and pulses, there is a little scale for escalating the area under fodder cultivation. Therefore, augment in the productivity of available forage resources per unit land area and time is necessitated.

Cowpea [Vigna unguiculata (L). Walp] is one of the most important legume forage crops. According to USDA food database, it has the highest percentage of calories from protein among vegetarian foods. In different countries, it is esteemed for its varied uses as food, green manure, nitrogen fixing (through root nodules) etc. As a forage crop, it is drought/shade tolerant, quick growing, high yielding, with substantially rich biomass production, grows well with associated crops. For fodder production, it is mainly grown as mixed/inter crop with cereals. Nevertheless, in forage legumes, quality seed production and availability is lacking.

Cowpea in Arunachal Pradesh is one of the important kharif forage legumes. The cultivation package accessible for its seed production is nearly lacking. Keeping this locale in view, the present research was intended with the following objectives.

1. To determine the optimum time of sowing, spacing and seed rate for higher seed production of fodder cowpea.

2. To study the quality parameters of seed and haulm. 


\section{Material And Methods}

The field experiment was conducted at Directorate of Animal Husb. \& Vety. Govt. of Arunachal Pradesh, Nirjuli,under rainfed conditions during kharif 2011.

The experimental soil site was medium deep black clay. The soil had medium $\left(285 \mathrm{~kg} \mathrm{ha}^{-1}\right)$ available nitrogen, available phosphorus $\left(21.68 \mathrm{~kg} \mathrm{ha}^{-1}\right)$ and available potassium $\left(208.53 \mathrm{~kg} \mathrm{ha}^{-1}\right)$.

\section{Experimental Details}

Treatments: 18 treatment combinations consisting of three times of sowing, two spacing and three seed rates were undertaken. The details of treatment are: A) Main plot-sowing time : June second fortnight; July first fortnight; July second fortnight B) Sub plot - Row spacing : $30 \mathrm{~cm}$; $45 \mathrm{~cm}$ C) Sub-sub plot - seed rate : 20 $\mathrm{kg} \mathrm{ha}^{-1} ; 25 \mathrm{~kg} \mathrm{ha}^{-1} ; 30 \mathrm{~kg} \mathrm{ha}^{-1}$

With three replications, the experiment was laid out in split - split plot design. The plot size was of Gross: $4.5 \mathrm{~m}$ x $4.0 \mathrm{~m} \&$ Net: $3.6 \mathrm{~m} \times 3.4 \mathrm{~m}$.

During 1988, Genotype 'Swad' (DFC-1) was released. With profuse branching, broad leaves and leafier, Plant grows to a height of $85 \mathrm{~cm}$, bushy \& compact. With maturity range of 85 days from seed to seed, it falls under medium maturity group. Seeds are generally dark brown and bold.

The statistics obtained by various observations were subjected to Fisher's method of analysis of variance and analysis of data as given by Gomez and Gomez (1984).

\section{Results \& Discussion}

The results obtained from the field experiment conducted at Directorate of Animal Husb. \& Vety. Govt. of Arunachal Pradesh, Nirjuli, during kharif 2011 to study the "impact of time of sowing, spacing and seed rate on potential seed production and fodder quality of cowpea" are discussed.

\section{Effect of Time of Sowing Growth and yield}

Significantly, delayed sowing reduced the yield of seed, haulm and haulm dry matter.

Compared to $\mathrm{D}_{2}$ viz., July first fortnight sowing $\left(673 \mathrm{~kg} \mathrm{ha}^{-1}\right)$ and $\mathrm{D}_{3}$ viz., July second fortnight sowing (517 kg ha- ), June second fortnight $\left(D_{1}\right)$ sowing produced significantly higher seed yield $\left(923 \mathrm{~kg} \mathrm{ha}^{-1}\right)$.

Though, $D_{2}$ was significant over $D_{3}$. $D_{1}$ produced 25 and 41 per cent higher grain yield compared to $D_{2}$ and $\mathrm{D}_{3}$, respectively.

Compared to $D_{2}$ and $D_{3}$, the haulm yield and harvest index were also significantly higher in $D_{1}$ (4440 $\mathrm{kg} \mathrm{ha}^{-1}$ and 0.3 , respectively). On the other hand, $\mathrm{D}_{2}$ was significant over $\mathrm{D}_{3}$.

Ravinder and Singh (1998) in mungbean, \& Yadav (2003) in cowpea, also reported similar results of higher seed yield in early sowing compared to late sowing.

Mainly due to significantly higher growth and yield components, there was increase in seed yield, haulm yield and harvest index with early sowing in June second fortnight $\left(D_{1}\right)$, compared to late sowing in $D_{2}$ and $\mathrm{D}_{3}$.

Significantly, June second fortnight sowing recorded higher plant height $(153.8 \mathrm{~cm}$, Table 3), number of leaves (36.4 plant ${ }^{-1}$, Table 4), number of branches $\left(5.0\right.$ plant $^{-1}$, Table 5), leaf area $\left(1048.98 \mathrm{~cm}^{2}\right.$ plant $^{-1}$, Table 6), LAI (2.19, Table 7), fresh weight (370.22 g plant ${ }^{-1}$, Table 8), total dry matter accumulation (63.32 $\mathrm{g} \mathrm{plant}^{-1}$, Table 9), number of pods (11.0 plant ${ }^{-1}$, Table 10), number of seeds (11.0 pod ${ }^{-1}$, Table 10) and 1000 seed weight $(100.0 \mathrm{~g}$, Table 10).

Table 1. Seed yield per plant, seed and seed dry matter yield per ha as influenced by time of sowing, row spacing and seed rate

\begin{tabular}{|c|c|c|c|c|c|c|c|c|c|c|}
\hline \multirow{3}{*}{$\begin{array}{l}\text { Time of } \\
\text { sowing }\end{array}$} & \multirow{3}{*}{$\begin{array}{l}\text { Seed rate } \\
\mathrm{kg} \mathrm{ha}^{-1}\end{array}$} & \multicolumn{6}{|c|}{ Seed yield (g plant-1) Seed yield (kg ha-1) } & \multicolumn{3}{|c|}{ Seed dry matter yield (kg ha-1) } \\
\hline & & \multicolumn{9}{|c|}{ Row spacing } \\
\hline & & $\begin{array}{l}\text { S1 (30 } \\
\mathrm{cm})\end{array}$ & $\mathrm{S} 2(45 \mathrm{~cm})$ & Mean & $\mathrm{S} 1(30 \mathrm{~cm})$ & $\mathrm{S} 2(45 \mathrm{~cm})$ & Mean & $\mathrm{S} 1(30 \mathrm{~cm})$ & $\mathrm{S} 2(45 \mathrm{~cm})$ & Mean \\
\hline \multirow{4}{*}{$\begin{array}{l}D_{1}-\text { Second } \\
\text { fortnight of } \\
\text { June }\end{array}$} & $\mathrm{P} 1-20$ & 14.33 & 13.67 & 14 & 933 & 805 & 869 & 845 & 729 & 787 \\
\hline & $\mathrm{P} 2-25$ & 12.28 & 12.71 & 12.5 & 1014 & 911 & 962 & 918 & 825 & 872 \\
\hline & P3-30 & 16.6 & 11.43 & 14 & 1054 & 823 & 938 & 954 & 745 & 850 \\
\hline & Mean & 14.4 & 12.6 & 13.5 & 1000 & 847 & 923 & 906 & 767 & 836 \\
\hline \multirow{4}{*}{$\begin{array}{l}\mathrm{D}_{2} \text { - First } \\
\text { fortnight of } \\
\text { July }\end{array}$} & P1-20 & 8.46 & 7.3 & 7.88 & 670 & 573 & 621 & 607 & 519 & 563 \\
\hline & $\mathrm{P} 2-25$ & 7.17 & 8.13 & 7.65 & 679 & 753 & 716 & 615 & 682 & 649 \\
\hline & \begin{tabular}{|l|} 
P3-30 \\
\end{tabular} & 7.19 & 7.4 & 7.29 & 754 & 610 & 682 & 683 & 552 & 618 \\
\hline & Mean & 7.61 & 7.61 & 7.61 & 701 & 645 & 673 & 635 & 584 & 610 \\
\hline \multirow{4}{*}{$\begin{array}{l}\mathrm{D}_{3} \text { - Second } \\
\text { fortnight of } \\
\text { July }\end{array}$} & P1-20 & 6.05 & 5.3 & 5.67 & 494 & 553 & 523 & 447 & 500 & 474 \\
\hline & $\mathrm{P} 2-25$ & 5.63 & 4.66 & 5.14 & 584 & 493 & 539 & 529 & 447 & 488 \\
\hline & P3-30 & 5.2 & 4.51 & 4.85 & 489 & 492 & 490 & 442 & 446 & 444 \\
\hline & Mean & 5.62 & 4.82 & 5.22 & 522 & 513 & 517 & 473 & 464 & 469 \\
\hline
\end{tabular}


Impact Of Time Of Sowing, Spacing And Seed Rate On Potential Seed Production And Fodder

\begin{tabular}{|l|l|l|l|l|l|l|l|l|l|l|}
\hline \multirow{6}{*}{} & P1-20 & 9.61 & 8.75 & 9.18 & 699 & 644 & 671 & 633 & 583 & 608 \\
\cline { 2 - 12 } & P2-25 & 8.36 & 8.5 & 8.43 & 759 & 719 & 739 & 687 & 651 & 669 \\
\cline { 2 - 12 } & P3-30 & 9.66 & 7.78 & 8.72 & 765 & 642 & 704 & 693 & 581 & 637 \\
\cline { 2 - 12 } & Mean & 9.24 & 8.34 & 8.78 & 741 & 668 & 705 & 671 & 605 & 638 \\
\hline
\end{tabular}

Table 2. Haulm, haulm dry matter yield and harvest index of cowpea as influenced by time of sowing, spacing and seed rate

\begin{tabular}{|c|c|c|c|c|c|c|c|c|c|c|}
\hline \multirow{3}{*}{$\begin{array}{l}\text { Time of } \\
\text { sowing }\end{array}$} & \multicolumn{3}{|c|}{ Seed rate Haulm yield $\left(\mathrm{kg} \mathrm{ha}^{-1}\right)$} & \multicolumn{7}{|c|}{ Haulm drymatter yield $\left(\mathrm{kg} \mathrm{ha}^{-1}\right)$ |Harvest index } \\
\hline & \multirow[t]{2}{*}{$\mathrm{kg} \mathrm{ha}^{-1}$} & \multicolumn{9}{|c|}{ Row spacing } \\
\hline & & $\mathrm{S}_{1}(30 \mathrm{~cm})$ & $\mathrm{S}_{2}(45 \mathrm{~cm})$ & Mean & $\mathrm{S}_{1}(30 \mathrm{~cm})$ & $\mathrm{S}_{2}(45 \mathrm{~cm})$ & Mean & $S_{1}(30 \mathrm{~cm})$ & $\mathrm{S}_{2}(45 \mathrm{~cm})$ & Mean \\
\hline \multirow{4}{*}{$\begin{array}{l}D_{1}-\text { Second } \\
\text { fortnight of } \\
\text { June }\end{array}$} & $\mathrm{P}_{1}-20$ & 4569 & 4244 & 4416 & 878 & 906 & 892 & 0.3 & 0.29 & 0.29 \\
\hline & $\mathrm{P}_{2}-25$ & 4838 & 4340 & 4589 & 852 & 708 & 780 & 0.31 & 0.31 & 0.31 \\
\hline & $\mathrm{P}_{3}-30$ & 4968 & 3661 & 4314 & 897 & 541 & 719 & 0.31 & 0.32 & 0.31 \\
\hline & Mean & 4791 & 4088 & 4440 & 876 & 718 & 797 & 0.3 & 0.3 & 0.3 \\
\hline \multirow{4}{*}{\begin{tabular}{|l}
$D_{2}-$ First \\
fortnight of \\
July
\end{tabular}} & $P_{1}-20$ & 4004 & 4286 & 4145 & 688 & 806 & 747 & 0.27 & 0.23 & 0.25 \\
\hline & $\mathrm{P}_{2}-25$ & 4070 & 4082 & 4076 & 708 & 712 & 710 & 0.26 & 0.28 & 0.27 \\
\hline & $\mathrm{P}_{3}-30$ & 4260 & 3455 & 3837 & 707 & 598 & 652 & 0.27 & 0.28 & 0.27 \\
\hline & Mean & 4111 & 3941 & 4026 & 701 & 705 & 703 & 0.27 & 0.26 & 0.26 \\
\hline \multirow{8}{*}{\begin{tabular}{|l}
$D_{3}$-Second \\
fortnight of \\
July
\end{tabular}} & $\mathrm{P}_{1}-20$ & 3757 & 3831 & 3794 & 690 & 651 & 671 & 0.23 & 0.24 & 0.24 \\
\hline & $\mathrm{P}_{2}-25$ & 3684 & 3633 & 3658 & 648 & 642 & 645 & 0.25 & 0.23 & 0.24 \\
\hline & $\mathrm{P}_{3}-30$ & 3614 & 3676 & 3645 & 603 & 603 & 603 & 0.23 & 0.23 & 0.23 \\
\hline & Mean & 3685 & 3713 & 3699 & 647 & 632 & 640 & 0.24 & 0.23 & 0.24 \\
\hline & $\mathrm{P}_{1}-20$ & 4110 & 4127 & 4118 & 752 & 788 & 770 & 0.26 & 0.25 & 0.26 \\
\hline & $\mathrm{P}_{2}-25$ & 4197 & 4018 & 4108 & 736 & 687 & 712 & 0.27 & 0.27 & 0.27 \\
\hline & $\mathrm{P}_{3}-30$ & 4280 & 3597 & 3939 & 735 & 581 & 658 & 0.27 & 0.27 & 0.27 \\
\hline & Mean & 4196 & 3914 & 4055 & 741 & 685 & 713 & 0.27 & 0.27 & 0.27 \\
\hline
\end{tabular}

Table 3. Plant height $(\mathrm{cm})$ of cowpea as influenced by time of sowing, spacing and seed rate

\begin{tabular}{|c|c|c|c|c|c|c|c|c|c|c|}
\hline \multirow{3}{*}{$\begin{array}{l}\text { Time of } \\
\text { sowing }\end{array}$} & \multirow{3}{*}{$\begin{array}{l}\text { Seed rate } \\
\mathrm{kg} \mathrm{ha}^{-1}\end{array}$} & \multicolumn{3}{|l|}{$30 \mathrm{DAS}$} & \multicolumn{3}{|c|}{60 DAS } & \multicolumn{2}{|c|}{ At harvest } & \\
\hline & & \multicolumn{9}{|c|}{ Row spacing } \\
\hline & & $\mathrm{S}_{1}(30 \mathrm{~cm})$ & $\mathrm{S}_{2}(45 \mathrm{~cm})$ & Mean & $\begin{array}{l}\mathrm{S}_{1}(30 \\
\mathrm{cm})\end{array}$ & $\mathrm{S}_{2}(45 \mathrm{~cm})$ & Mean & $\mathrm{S}_{1}(30 \mathrm{~cm})$ & $\mathrm{S}_{2}(45 \mathrm{~cm})$ & M ean \\
\hline \multirow{4}{*}{$\begin{array}{l}D_{1} \text { - Second } \\
\text { fortnight of } \\
\text { June }\end{array}$} & $\mathrm{P}_{1}-20$ & 20.1 & 18.7 & 19.4 & 56.4 & 54.4 & 55.4 & 156.8 & 150.8 & 153.8 \\
\hline & $\mathrm{P}_{2}-25$ & 20.7 & 19.2 & 19.9 & 56.8 & 52.7 & 54.8 & 166.7 & 148.7 & 157.7 \\
\hline & $\mathrm{P}_{3}-30$ & 21.2 & 20.6 & 20.9 & 51.9 & 42.5 & 47.2 & 154.4 & 145.4 & 149.9 \\
\hline & Mean & 20.6 & 19.5 & 20.1 & 55.0 & 49.9 & 52.5 & 159.1 & 148.3 & 153.8 \\
\hline \multirow{4}{*}{$\begin{array}{l}D_{2} \text {-First } \\
\text { fortnight of } \\
\text { July }\end{array}$} & $P_{1}-20$ & 13.3 & 14.1 & 13.7 & 36.2 & 34.6 & 35.4 & 140.6 & 134.7 & 137.7 \\
\hline & $\mathrm{P}_{2}-25$ & 14.9 & 12.5 & 13.7 & 37.5 & 36.5 & 37.0 & 147.1 & 134.7 & 140.9 \\
\hline & $\mathrm{P}_{3}-30$ & 14.5 & 14.2 & 14.3 & 35.0 & 32.7 & 33.8 & 143.3 & 133.3 & 138.3 \\
\hline & Mean & 14.2 & 13.6 & 13.9 & 36.2 & 34.6 & 35.4 & 143.7 & 134.3 & 139.0 \\
\hline \multirow{8}{*}{$\begin{array}{l}\mathrm{D}_{3} \text { - Second } \\
\text { fortnight of } \\
\text { July }\end{array}$} & $P_{1}-20$ & 12.6 & 12.6 & 12.6 & 37.1 & 36.4 & 36.8 & 139.7 & 138.1 & 138.9 \\
\hline & $\mathrm{P}_{2}-25$ & 12.5 & 12.1 & 12.3 & 37.1 & 31.7 & 33.4 & 138.1 & 135.4 & 136.7 \\
\hline & $\mathrm{P}_{3}-30$ & 11.0 & 12.2 & 11.6 & 35.2 & 34.7 & 34.9 & 138.1 & 133.1 & 135.6 \\
\hline & Mean & 12.0 & 12.3 & 12.2 & 35.8 & 34.3 & 35.0 & 138.6 & 135.5 & 137.1 \\
\hline & $\mathrm{P}_{1}-20$ & 15.3 & 15.1 & 15.2 & 43.2 & 41.8 & 42.5 & 145.7 & 141.2 & 143.4 \\
\hline & $P_{2}-25$ & 16.0 & 14.6 & 15.3 & 43.1 & 40.3 & 41.7 & 150.6 & 139.6 & 145.1 \\
\hline & $\mathrm{P}_{3}-30$ & 15.5 & 15.6 & 15.6 & 40.7 & 36.6 & 38.6 & 145.3 & 137.3 & 141.3 \\
\hline & Mean & 15.6 & 15.1 & 15.4 & 42.3 & 39.6 & 41.0 & 147.2 & 139.3 & 143.3 \\
\hline
\end{tabular}

Table 4. Number of green trifoliate leaves $\left(\right.$ plant $^{-1}$ ) of cowpea as influenced by time of sowing, spacing and seed rate

\begin{tabular}{|c|c|c|c|c|c|c|c|c|c|c|}
\hline \multirow{3}{*}{$\begin{array}{l}\text { Time of } \\
\text { sowing }\end{array}$} & \multirow{3}{*}{$\begin{array}{l}\text { Seed rate } \\
\mathrm{kg} \mathrm{ha}^{-1}\end{array}$} & \multicolumn{9}{|c|}{30 DAS 60 DAS At harvest } \\
\hline & & \multicolumn{9}{|c|}{ Row spacing } \\
\hline & & $\mathrm{S} 1(30 \mathrm{~cm})$ & $\mathrm{S} 2(45 \mathrm{~cm})$ & Mean & $\mathrm{S} 1(30 \mathrm{~cm})$ & $\mathrm{S} 2(45 \mathrm{~cm})$ & Mean & $\mathrm{S} 1(30 \mathrm{~cm})$ & $\begin{array}{l}\mathrm{S} 2(45 \\
\mathrm{cm})\end{array}$ & Mean \\
\hline \multirow{4}{*}{$\begin{array}{l}\mathrm{D}_{1} \text { - Second } \\
\text { fortnight of } \\
\text { June }\end{array}$} & $P_{1}-20$ & 3.8 & 3.4 & 3.6 & 28.16 & 25.73 & 26.95 & 38.50 & 34.43 & 36.46 \\
\hline & $\mathrm{P}_{2}-25$ & 6.4 & 4.5 & 5.5 & 29.00 & 25.06 & 27.03 & 40.43 & 35.13 & 37.78 \\
\hline & $\mathrm{P}_{3}-30$ & 4.9 & 6.6 & 5.7 & 27.00 & 24.00 & 25.50 & 36.33 & 33.80 & 35.06 \\
\hline & Mean & 5.0 & 4.8 & 4.9 & 28.05 & 24.93 & 26.49 & 38.42 & 34.45 & 36.43 \\
\hline \multirow{4}{*}{$\begin{array}{l}\mathrm{D}_{2} \text { - First } \\
\text { fortnight of } \\
\text { July }\end{array}$} & $P_{1}-20$ & 3.0 & 4.3 & 3.6 & 25.53 & 19.83 & 22.68 & 29.43 & 28.76 & 29.10 \\
\hline & $\mathrm{P}_{2}-25$ & 3.5 & 3.6 & 3.5 & 23.43 & 20.80 & 22.11 & 29.10 & 29.10 & 29.10 \\
\hline & $\mathrm{P}_{3}-30$ & 3.8 & 3.5 & 3.6 & 22.76 & 19.53 & 21.15 & 28.76 & 28.76 & 28.76 \\
\hline & Mean & 3.4 & 3.8 & 3.6 & 23.91 & 20.05 & 21.98 & 29.10 & 28.87 & 28.98 \\
\hline \multirow{8}{*}{$\begin{array}{l}\mathrm{D}_{3} \text { - Second } \\
\text { fortnight of } \\
\text { July }\end{array}$} & $P_{1-20}$ & 3.6 & 3.2 & 3.4 & 22.23 & 22.00 & 22.11 & 29.76 & 28.43 & 29.10 \\
\hline & $P_{2}-25$ & 3.6 & 3.3 & 3.4 & 22.73 & 20.06 & 21.40 & 28.76 & 28.10 & 28.43 \\
\hline & $P_{3}-30$ & 2.9 & 2.3 & 2.6 & 22.80 & 19.80 & 21.30 & 30.76 & 27.10 & 28.93 \\
\hline & Mean & 3.4 & 2.9 & 3.1 & 22.58 & 20.62 & 21.60 & 29.76 & 27.87 & 28.82 \\
\hline & $P_{1}-20$ & 3.5 & 3.6 & 4.0 & 25.31 & 22.52 & 23.91 & 32.56 & 30.54 & 31.55 \\
\hline & $P_{2}-25$ & 4.5 & 3.8 & 4.1 & 25.05 & 21.97 & 23.51 & 32.76 & 30.77 & 31.77 \\
\hline & $P_{3}-30$ & 3.8 & 4.1 & 4.0 & 24.18 & 21.11 & 22.65 & 31.95 & 29.88 & 30.92 \\
\hline & Mean & 3.9 & 3.8 & 3.9 & 24.85 & 21.87 & 23.36 & 32.43 & 30.40 & 31.41 \\
\hline
\end{tabular}


Table 5. Number of branches (plant ${ }^{-1}$ ) of cowpea as influenced by time of sowing, spacing and seed rate

\begin{tabular}{|c|c|c|c|c|c|c|c|}
\hline \multirow{3}{*}{$\begin{array}{l}\text { Time of } \\
\text { sowing }\end{array}$} & \multirow{3}{*}{$\begin{array}{l}\text { Seed rate } \\
\mathrm{kg} \mathrm{ha}^{-1}\end{array}$} & \multicolumn{3}{|c|}{60 DAS } & \multicolumn{3}{|c|}{ At harvest } \\
\hline & & \multicolumn{6}{|c|}{ Row spacing } \\
\hline & & $\begin{array}{l}\mathrm{S}_{1}(30 \\
\mathrm{cm})\end{array}$ & $\begin{array}{l}\mathrm{S}_{2}(45 \\
\mathrm{cm})\end{array}$ & Mea & $\mathrm{S}_{1}(30 \mathrm{~cm})$ & $\mathrm{S}_{2}(45 \mathrm{~cm})$ & Mean \\
\hline \multirow{4}{*}{$\begin{array}{l}D_{1} \text { - Second } \\
\text { fortnight of } \\
\text { June }\end{array}$} & $P_{1}-20$ & 4.3 & 3.9 & 4.1 & 5.9 & 4.9 & 5.4 \\
\hline & $\mathrm{P}_{2}-25$ & 4.9 & 3.7 & 4.3 & 6.4 & 3.7 & 5.1 \\
\hline & $\mathrm{P}_{3}-30$ & 4.2 & 4.3 & 4.2 & 5.3 & 4.1 & 4.7 \\
\hline & Mean & 4.5 & 4 & 4.2 & 5.8 & 4.2 & 5 \\
\hline \multirow{4}{*}{$\begin{array}{l}\mathrm{D}_{2} \text { - First } \\
\text { fortnight of } \\
\text { July }\end{array}$} & $P_{1}-20$ & 3.1 & 1.5 & 2.3 & 4.3 & 2.1 & 3.2 \\
\hline & $P_{2}-25$ & 2.2 & 1.9 & 2.1 & 3.1 & 2.9 & 3 \\
\hline & $\mathrm{P}_{3}-30$ & 1.9 & 2.2 & 2 & 2.8 & 2.6 & 2.7 \\
\hline & Mean & 2.4 & 1.9 & 2.1 & 3.4 & 2.5 & 3 \\
\hline \multirow{8}{*}{$\begin{array}{l}\mathrm{D}_{3} \text {-Second } \\
\text { fortnight of } \\
\text { July }\end{array}$} & $\mathrm{P}_{1}-20$ & 2.4 & 1.8 & 2.1 & 2.9 & 2.7 & 2.8 \\
\hline & $\mathrm{P}_{2}-25$ & 1.8 & 1.7 & 1.8 & 2.1 & 3.4 & 2.8 \\
\hline & $\mathrm{P}_{3}-30$ & 2 & 1.5 & 1.8 & 3.2 & 2.9 & 3 \\
\hline & Mean & 2.1 & 1.7 & 1.9 & 2.7 & 3 & 2.9 \\
\hline & $P_{1}-20$ & 3.3 & 2.4 & 2.8 & 4.4 & 3.2 & 3.8 \\
\hline & $P_{2}-25$ & 3 & 2.4 & 2.7 & 3.9 & 3.3 & 3.6 \\
\hline & $\mathrm{P}_{3}-30$ & 2.7 & 2.7 & 2.7 & 3.8 & 3.2 & 3.5 \\
\hline & Mean & 3.0 & 2.5 & 2.7 & 4.0 & 3.2 & 3.6 \\
\hline
\end{tabular}

Table 6. Leaf area $\left(\mathrm{cm}^{2}\right.$ plant $\left.{ }^{-1}\right)$ of cowpea as influenced by time of sowing, spacing and seed rate

\begin{tabular}{|c|c|c|c|c|c|c|c|c|c|c|}
\hline \multirow{3}{*}{$\begin{array}{l}\text { Time of } \\
\text { sowing }\end{array}$} & \multirow{3}{*}{$\begin{array}{l}\text { Seed rate } \\
\mathrm{kg} \mathrm{ha}^{-1}\end{array}$} & $30 \mathrm{DAS}$ & & & \multicolumn{3}{|l|}{60 DAS } & \multicolumn{3}{|l|}{ At harvest } \\
\hline & & \multicolumn{9}{|c|}{ Row spacing } \\
\hline & & $\mathrm{S}_{1}(30 \mathrm{~cm})$ & $\mathrm{S}_{2}(45 \mathrm{~cm})$ & Mean & $\mathrm{S}_{1}(30 \mathrm{~cm})$ & $\mathrm{S}_{2}(45 \mathrm{~cm})$ & Mean & $\mathrm{S}_{1}(30 \mathrm{~cm})$ & $\mathrm{S}_{2}(45 \mathrm{~cm}$ & Mean \\
\hline \multirow{4}{*}{\begin{tabular}{|l|}
$D_{1^{-}}$ \\
Second \\
fortnight \\
of June \\
\end{tabular}} & $P_{1}-20$ & 236.03 & 239.9 & 237.96 & 1019.43 & 865.2 & 942.31 & 1243.74 & 1102.91 & 1173.32 \\
\hline & $P_{2}-25$ & 266.93 & 270.7 & 268.81 & 990.1 & 905.73 & 947.91 & 1079.62 & 913.99 & 986.8 \\
\hline & $P_{3}-30$ & 241.5 & 280.73 & 261.11 & 1038 & 879.23 & 958.61 & 1012.67 & 940.94 & 976.8 \\
\hline & Mean & 248.15 & 263.77 & 255.96 & 1015.84 & 883.38 & 949.61 & 1112.01 & 985.95 & 1048.98 \\
\hline \multirow{4}{*}{$\begin{array}{l}\mathrm{D}_{2} \text { - First } \\
\text { fortnight } \\
\text { of July }\end{array}$} & $P_{1}-20$ & 210.6 & 248.76 & 229.68 & 952.53 & .33 & 779.93 & .82 & 61 & 855.72 \\
\hline & $P_{2}-25$ & 205.53 & 225.93 & 215.73 & 609.12 & 741.56 & 675.34 & 998.25 & 824.81 & 911.53 \\
\hline & $\mathrm{P}_{3}-30$ & 219.46 & 201.23 & 210.35 & 730.83 & 659.8 & 695.31 & 987.7 & .24 & 850.47 \\
\hline & Mean & 211.86 & 225.31 & 218.58 & 764.16 & 669.56 & 716.86 & 1000.26 & 744.89 & 872.57 \\
\hline \multirow{8}{*}{$\begin{array}{l}\mathrm{D}_{3^{-}} \\
\text {Second } \\
\text { fortnight } \\
\text { of July }\end{array}$} & $P_{1}-20$ & 213.8 & 214.63 & 214.21 & 853.7 & 647.83 & 750.76 & 912.04 & 775.72 & 843.88 \\
\hline & $P_{2}-25$ & 226.06 & 239.33 & 232.7 & 796.56 & .73 & 670.65 & .31 & 784 & 818.66 \\
\hline & $\mathrm{P}_{3}-30$ & 227.3 & 194.66 & 210.98 & 728.63 & 522.86 & 625.75 & 932.12 & 601.59 & 766.85 \\
\hline & Mean & 222.38 & 216.21 & 219.3 & 792.96 & 571.81 & 682.38 & 899.16 & 720.44 & 809.8 \\
\hline & $P_{1}-20$ & 220.14 & 234.43 & 227.28 & 941.88 & 706.78 & 824.33 & 1056.87 & 858.41 & 957.64 \\
\hline & $P_{2}-25$ & 232.84 & 245.32 & 239.08 & 798.59 & 730.67 & 764.63 & 977.06 & 840.93 & 909 \\
\hline & $\mathrm{P}_{3}-30$ & 229.42 & 225.54 & 227.48 & 832.48 & 687.3 & 759.89 & 977.49 & 751.92 & 864.71 \\
\hline & Mean & 227.47 & 235.10 & 231.28 & 857.65 & 708.25 & 782.95 & 1003.81 & 817.09 & 909.78 \\
\hline
\end{tabular}

Table 7. Leaf area index (LAI) of cowpea as influenced by time of sowing, spacing and seed rate

\begin{tabular}{|c|c|c|c|c|c|c|c|c|c|c|}
\hline \multirow{3}{*}{$\begin{array}{l}\text { Time of } \\
\text { sowing }\end{array}$} & \multirow{3}{*}{$\begin{array}{l}\text { Seed rate } \\
\mathrm{kg} \text { ha-1 }\end{array}$} & 30 DAS & & \multicolumn{3}{|c|}{60 DAS } & \multicolumn{4}{|c|}{ At harvest } \\
\hline & & \multicolumn{9}{|c|}{ Row spacing } \\
\hline & & $\mathrm{S} 1(30 \mathrm{~cm})$ & $\mathrm{S} 2(45 \mathrm{~cm})$ & Mean & $\mathrm{S} 1(30 \mathrm{~cm})$ & $\mathrm{S} 2(45 \mathrm{~cm})$ & Mean & $\mathrm{S} 1(30 \mathrm{~cm})$ & $\mathrm{S} 2(45 \mathrm{~cm})$ & Mean \\
\hline \multirow{4}{*}{$\begin{array}{l}\text { D1- Second } \\
\text { fortnight of } \\
\text { June }\end{array}$} & $P_{1}-20$ & 0.53 & 0.54 & 0.53 & 1.89 & 1.62 & 1.75 & 2.11 & 1.88 & 1.99 \\
\hline & $\mathrm{P}_{2}-25$ & 0.71 & 0.71 & 0.71 & 2.25 & 2.07 & 2.16 & 2.26 & 1.93 & 2.09 \\
\hline & $\mathrm{P}_{3}-30$ & 0.77 & 0.88 & 0.83 & 2.83 & 2.41 & 2.62 & 2.57 & 2.39 & 2.48 \\
\hline & Mean & 0.67 & 0.71 & 0.69 & 2.32 & 2.03 & 2.18 & 2.31 & 2.06 & 2.19 \\
\hline \multirow{4}{*}{$\begin{array}{l}\text { D2- First } \\
\text { fortnight of } \\
\text { July }\end{array}$} & $\mathrm{P}_{1}-20$ & 0.48 & 0.55 & 0.52 & 1.77 & 1.16 & 1.47 & 1.74 & 1.22 & 1.48 \\
\hline & $\mathrm{P}_{2}-25$ & 0.57 & 0.62 & 0.59 & 1.43 & 1.72 & 1.57 & 2.1 & 1.75 & 1.92 \\
\hline & $\mathrm{P}_{3}-30$ & 0.71 & 0.66 & 0.69 & 2.02 & 1.83 & 1.93 & 2.51 & 1.84 & 2.18 \\
\hline & Mean & 0.59 & 0.61 & 0.6 & 1.74 & 1.57 & 1.65 & 2.11 & 1.6 & 1.86 \\
\hline \multirow{8}{*}{$\begin{array}{l}\text { D3- Second } \\
\text { fortnight of } \\
\text { July }\end{array}$} & $\mathrm{P}_{1}-20$ & 0.49 & 0.49 & 0.49 & 1.6 & 1.24 & 1.42 & 1.57 & 1.35 & 1.46 \\
\hline & $\mathrm{P}_{2}-25$ & 0.62 & 0.64 & 0.63 & 1.83 & 1.29 & 1.56 & 1.81 & 1.67 & 1.74 \\
\hline & $P_{3}-30$ & 0.74 & 0.64 & 0.69 & 2.02 & 1.48 & 1.75 & 2.37 & 1.57 & 1.97 \\
\hline & Mean & 0.61 & 0.59 & 0.6 & 1.82 & 1.33 & 1.57 & 1.92 & 1.53 & 1.72 \\
\hline & $\mathrm{P}_{1}-20$ & 0.5 & 0.53 & 0.51 & 1.66 & 1.34 & 1.54 & 1.8 & 1.48 & 1.64 \\
\hline & $\mathrm{P}_{2}-25$ & 0.63 & 0.66 & 0.64 & 1.84 & 1.69 & 1.76 & 2.05 & 1.78 & 1.92 \\
\hline & $P_{3}-30$ & 0.74 & 0.73 & 0.73 & 2.29 & 1.91 & 2.1 & 2.48 & 1.93 & 2.21 \\
\hline & Mean & 0.62 & 0.64 & 0.63 & 1.96 & 1.65 & 1.80 & 2.11 & 1.73 & 1.92 \\
\hline
\end{tabular}


Table 8. Fresh weight $\left(\mathrm{g} \mathrm{plant}^{-1}\right)$ of cowpea as influenced by time of sowing, spacing and seed rate

\begin{tabular}{|c|c|c|c|c|c|c|c|c|c|c|}
\hline \multirow{3}{*}{$\begin{array}{l}\text { Time of } \\
\text { sowing }\end{array}$} & \multirow{3}{*}{$\begin{array}{l}\text { Seed } \\
\text { rate }\end{array}$} & \multicolumn{3}{|c|}{30 DAS } & \multicolumn{2}{|c|}{60 DAS } & \multicolumn{4}{|c|}{ At harvest } \\
\hline & & \multicolumn{9}{|c|}{ Row spacing } \\
\hline & & $\begin{array}{l}\mathrm{S}_{1}(30 \\
\mathrm{cm})\end{array}$ & $\begin{array}{l}\mathrm{S}_{2}(45 \\
\mathrm{cm})\end{array}$ & Mean & $\begin{array}{l}\mathrm{S}_{1}(30 \\
\mathrm{cm})\end{array}$ & $\begin{array}{l}\mathrm{S}_{2}(45 \\
\mathrm{cm})\end{array}$ & Mean & $\begin{array}{l}\mathrm{S}_{1}(30 \\
\mathrm{cm})\end{array}$ & $\begin{array}{l}\mathrm{S}_{2}(45 \\
\mathrm{cm})\end{array}$ & Mean \\
\hline \multirow{4}{*}{$\begin{array}{l}\mathrm{D}_{1-} \\
\text { Second } \\
\text { fortnight } \\
\text { of June }\end{array}$} & $\mathrm{P}_{1}-20$ & 16.82 & 15.3 & 16.06 & 132.33 & 123.76 & 128.05 & 394.96 & 346.43 & 370.69 \\
\hline & $\mathrm{P}_{2}-25$ & 16.5 & 17.2 & 16.85 & 133.96 & 114.76 & 124.36 & 389.04 & 351.43 & 370.23 \\
\hline & $\mathrm{P}_{3}-30$ & 17.7 & 17.24 & 17.47 & 132 & 114.56 & 123.28 & 355.12 & 384.37 & 369.74 \\
\hline & Mean & 17 & 16.58 & 16.79 & 132.76 & 117.7 & 125.23 & 379.7 & 360.74 & 370.22 \\
\hline \multirow{4}{*}{$\begin{array}{l}\mathrm{D}_{2} \text { - First } \\
\text { fortnight } \\
\text { of July }\end{array}$} & $\mathrm{P}_{1}-20$ & 12.72 & 15.46 & 14.09 & 116.5 & 118.43 & 117.46 & 359.93 & 281.73 & 320.83 \\
\hline & $\mathrm{P}_{2}-25$ & 12 & 11.43 & 11.72 & 131.13 & 107.76 & 119.45 & 331.77 & 298.37 & 315.07 \\
\hline & $\mathrm{P}_{3}-30$ & 13.08 & 8.76 & 10.92 & 96.6 & 96.93 & 96.76 & 326.68 & 298.36 & 312.52 \\
\hline & Mean & 12.6 & 11.88 & 12.24 & 114.74 & 107.71 & 111.22 & 339.46 & 292.82 & 316.14 \\
\hline \multirow{8}{*}{$\begin{array}{l}\mathrm{D}_{3^{-}} \\
\text {Second } \\
\text { fortnight } \\
\text { of July }\end{array}$} & $P_{1}-20$ & 14.79 & 13.4 & 14.1 & 108.26 & 106.76 & 107.51 & 305.44 & 305.16 & 305.3 \\
\hline & $\mathrm{P}_{2}-25$ & 17.2 & 16.58 & 16.89 & 108.6 & 108.1 & 108.35 & 311.78 & 305.08 & 308.43 \\
\hline & $\mathrm{P}_{3}-30$ & 14.38 & 10.3 & 12.34 & 104.93 & 107.26 & 106.1 & 321.68 & 308.44 & 315.06 \\
\hline & Mean & 15.46 & 13.43 & 14.44 & 107.26 & 107.37 & 107.32 & 312.97 & 306.23 & 309.6 \\
\hline & $\mathrm{P}_{1}-20$ & 14.78 & 14.72 & 14.75 & 119.03 & 116.32 & 117.67 & 353.44 & 311.11 & 332.27 \\
\hline & $\mathrm{P}_{2}-25$ & 15.23 & 15.07 & 15.15 & 124.56 & 110.21 & 117.68 & 344.19 & 318.29 & 331.24 \\
\hline & $\mathrm{P}_{3}-30$ & 15.05 & 12.1 & 13.58 & 111.17 & 106.25 & 108.71 & 334.49 & 330.39 & 332.44 \\
\hline & Mean & 14.39 & 13.96 & 14.49 & 118.15 & 110.93 & 114.59 & 344.04 & 319.93 & 331.99 \\
\hline
\end{tabular}

Table 9. Total dry matter accumulation $\left(\mathrm{g} \mathrm{plant}^{-1}\right)$ of cowpea as influenced by time of sowing, spacing and seed rate

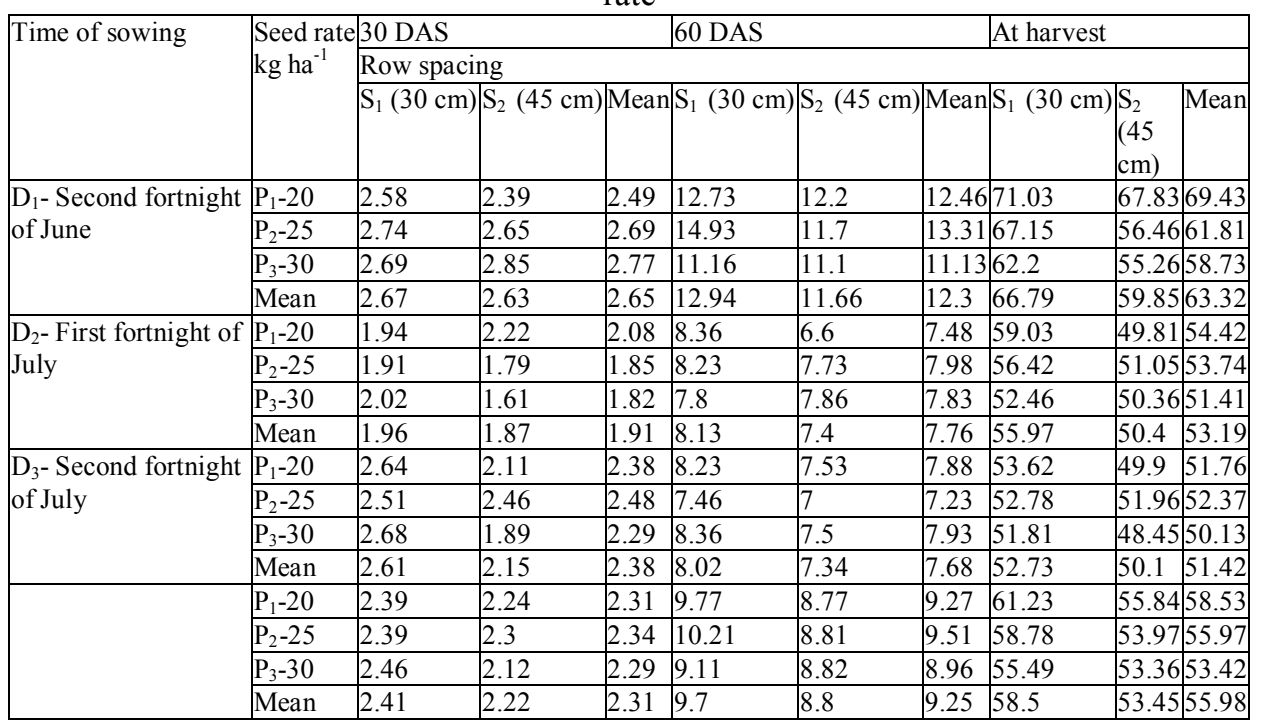

Table 10. Number of pods per plant, number of seeds per pod and 1000 seed weight of cowpea as influenced by time of sowing, spacing and seed rate at harvest.

\begin{tabular}{|c|c|c|c|c|c|c|c|c|c|c|}
\hline \multirow{3}{*}{$\begin{array}{l}\text { Time of } \\
\text { sowing }\end{array}$} & \multirow{3}{*}{$\begin{array}{l}\text { Seed rat } \\
\mathrm{kg} \mathrm{ha}^{-1}\end{array}$} & \multirow{2}{*}{\multicolumn{3}{|c|}{\begin{tabular}{|l|} 
Number of poc \\
Row spacing \\
\end{tabular}}} & \multicolumn{3}{|c|}{ Number of seeds $\left(\mathrm{pod}^{-1}\right)$} & \multicolumn{3}{|c|}{1000 seed weight $(\mathrm{g})$} \\
\hline & & & & & & & & & & \\
\hline & & $\mathrm{S}_{1}(30 \mathrm{~cm})$ & $\mathrm{S}_{2}(45 \mathrm{~cm})$ & Mean & $\mathrm{S}_{1}(30 \mathrm{~cm})$ & $\mathrm{S}_{2}(45 \mathrm{~cm})$ & Mear & $\mathrm{S}_{1}(30 \mathrm{~cm})$ & $\mathrm{S}_{2}(45 \mathrm{~cm})$ & Mean \\
\hline \multirow{4}{*}{$\begin{array}{l}\mathrm{D}_{1^{-}} \\
\text {Second } \\
\text { fortnight } \\
\text { of June }\end{array}$} & $P_{1}-20$ & 11.7 & 10.7 & 11.2 & 11.3 & 11.2 & 11.3 & 99.4 & 99.3 & 99.3 \\
\hline & $\mathrm{P}_{2}-25$ & 11.4 & 9.8 & 10.6 & 10.5 & 10.3 & 10.4 & 100.6 & 99.2 & 99.9 \\
\hline & $\mathrm{P}_{3}-30$ & 12.3 & 9.9 & 11.1 & 12.1 & 10.5 & 11.3 & 101.4 & 100.4 & 100.9 \\
\hline & Mean & 11.8 & 10.1 & 11 & 11.3 & 10.7 & 11 & 100.5 & 99.6 & 100 \\
\hline \multirow{4}{*}{$\begin{array}{l}\mathrm{D}_{2} \text { - First } \\
\text { fortnight } \\
\text { of July }\end{array}$} & $P_{1}-20$ & 8 & 8.1 & 8.1 & 9.1 & 9.6 & 9.3 & 96.6 & 96.9 & 96.7 \\
\hline & $P_{2}-25$ & 8.6 & 8.5 & 8.6 & 9.4 & 9.3 & 9.3 & 96.9 & 97 & 97 \\
\hline & $\mathrm{P}_{3}-30$ & 8.7 & 8.2 & 8.4 & 9.1 & 9.3 & 9.2 & 97.3 & 96.9 & 97.1 \\
\hline & Mean & 8.4 & 8.3 & 8.3 & 9.2 & 9.4 & 9.3 & 96.9 & 97 & 96.9 \\
\hline \multirow{8}{*}{$\begin{array}{l}\mathrm{D}_{3}- \\
\text { Second } \\
\text { fortnigh } \\
\text { of July }\end{array}$} & $P_{1}-20$ & 6.2 & 6.9 & 6.5 & 9.1 & 9.4 & 9.2 & 96.7 & 96.4 & 96.5 \\
\hline & $P_{2}-25$ & 6.9 & 6.7 & 6.8 & 8.8 & 9.4 & 9.1 & 96.5 & 96.2 & 96.4 \\
\hline & $P_{3}-30$ & 7.6 & 6.2 & 6.9 & 9.4 & 9.4 & 9.4 & 96.7 & 96.8 & 96.7 \\
\hline & Mean & 6.9 & 6.6 & 6.7 & 9.1 & 9.4 & 9.2 & 96.6 & 96.5 & 96.5 \\
\hline & $P_{1}-20$ & 8.6 & 8.6 & 8.6 & 9.8 & 10.1 & 9.9 & 97.5 & 97.5 & 97.5 \\
\hline & $\mathrm{P}_{2}-25$ & 9 & 8.3 & 8.6 & 9.5 & 9.6 & 9.6 & 98 & 97.5 & 97.7 \\
\hline & $P_{3}-30$ & 9.5 & 8.1 & 8.8 & 10.2 & 9.7 & 10 & 98.5 & 98 & 98.2 \\
\hline & Mean & 9 & 8.3 & 8.7 & 9.8 & 9.8 & 9.8 & 98 & 97.7 & 97.8 \\
\hline
\end{tabular}


These findings are in consistency with the findings of Rutkowski and Fordonski (1987) for plant height and dry matter accumulation, Kurmawanshi et al. (1994) for plant height and branches per plant in field bean, Sreelatha et al. (1997) for dry matter production, leaf area and leaf area index and Begum et al. (2003) for plant height and leaf area in french bean with early sowing, Ravinder and Singh (1998) for plant height, Yadav (2003) for plant height, number of pods and test weight in cowpea.

The photosynthetic area and activity of the crop leading to better growth and yield components contributing to more seed yield might have been increased by significantly higher number of leaves, leaf area and LAI In early sowing.

In delayed sowing viz., second fortnight of July $\left(\mathrm{D}_{3}\right)$, due to the significantly lowest growth and yield components, especially leaf area and leaf area index, which might have affected the photosynthesis and intern the yield, significantly least seed yield (517 $\mathrm{kg} \mathrm{ha}^{-1}$, Table 1$)$, haulm yield (3699 $\mathrm{kg} \mathrm{ha}^{-1}$, Table 2$)$ and harvest index $(0.24$, Table 2$)$ recorded.

\section{Proximate Composition (quality) Seed}

The contents of seed quality parameters were not affected significantly, irrespective of time of sowing. On the other hand, the crude protein content ranged from 21.97 to 22.92 per cent.

Mainly due to significantly higher seed dry matter yield recorded with $D_{1}$ compared to $D_{2}$ and $D_{3}$, the yield of all the seed quality parameters were significantly higher in June second fortnight sowing $\left(\mathrm{D}_{1}\right)$ compared to $\mathrm{D}_{2}$ and $\mathrm{D}_{3}$.

Due to significantly lowest seed dry matter yield, the significantly lowest yield of all the quality parameters recorded in $\mathrm{D}_{3}$.

\section{Haulm}

Time of sowing did not significantly influence the content and yield of all the quality parameters. Mainly due to the on par haulm dry matter yield recorded for time of sowing, the non-significant yield of quality parameters were recorded.

\section{Seed Quality}

signifying that seed quality is not affected by time of sowing, time of sowing did not significantly influence the seed quality parameters namely germination percentage (87.6 to $89.0 \%$ ), field emergence, root length, shoot length, total seedling length $(32.4 \mathrm{~cm}$ to $34.3 \mathrm{~cm}$ ) and seedling vigour index (3043.7 to 3264.4).

For germination percentage in Pea, these result are in concord with Hari and Gill (1981).

\section{Effect Of Spacing}

\section{Growth and yield}

Compared to wider row spacing of $45 \mathrm{~cm}$, the closer row spacing of $30 \mathrm{~cm}$ produced significantly higher seed yield $\left(741 \mathrm{~kg} \mathrm{ha}^{-1}\right)$ and haulm yield $\left(4196 \mathrm{~kg} \mathrm{ha}^{-1}\right)$, which accounted 9 per cent and 5 per cent higher, respectively (Table 1, 2).

Mainly due to significantly higher performance of all the growth and yield components, there was increase in seed and haulm yield with $30 \mathrm{~cm}$ row spacing compared to $45 \mathrm{~cm}$.

In closer spacing, compared to wider spacing, these findings are in consistency with Mc Ewen (1973) in field bean, Dwivedi et al. (1994), Singh and Tripathi (1994) in French bean, Angne et al. (1993), Arora et al. (1971) and Yadav (2003) in cowpea.

Significantly higher plant height $\left(147.2 \mathrm{~cm}\right.$, Table 3), number of green trifoliate leaves (32.4 plant ${ }^{-1}$, Table 4), number of branches (4.0 plant ${ }^{-1}$, Table5), leaf area $\left(1003.81 \mathrm{~cm}^{2}\right.$ plant $^{-1}$, Table 6$)$, leaf area index (2.11, Table 7), fresh weight (344.04 $\mathrm{g} \mathrm{plant}^{-1}$, Table 8), total dry matter accumulation (58.50 g plant ${ }^{-1}$, Table 9), number of pods $\left(9.0\right.$ plant $^{-1}$, Table 10) and seed yield $\left(09.24 \mathrm{~g}\right.$ plant $^{-1}$, Table 1) were recorded in $30 \mathrm{~cm}$ row spacing.

These findings are in consistency with the result of Angne et al. (1993) for growth parameters and Arora et al. (1971) for plant height, lateral branches and number of trifoliate leaves in cowpea, Mc Ewen (1973) for plant height in field bean, Singh and Tripathi (1994) for plant height, branches and leaves per plant and Dwivedi et al. (1995) for plant height, number of leaves and branches per plant in french bean and Yadav (2003) for plant height.

Significantly higher number of leaves, leaf area and leaf area index which might have increased the photosynthetic area and activity of the crop leading to better growth and yield components contributing to more seed yield recorded in row spacing of $30 \mathrm{~cm}$.

Due to the significantly lowest growth and yield components, significantly least seed yield (668 $\mathrm{kg} \mathrm{ha}^{-}$ ${ }^{1}$ ) and haulm yield (3914 $\left.\mathrm{kg} \mathrm{ha}^{-1}\right)$ were recorded with $45 \mathrm{~cm}$ row spacing. 


\section{Proximate composition (quality) Seed}

These outcomes are in agreement with Rajesh et al. (1998). Row spacing did not significantly influence the content and yield of all the quality parameters. Mainly due to the on par seed dry matter yield recorded for row spacing, the non-significant yield of quality parameters was recorded. On the other hand, crude protein content ranged from 22.52 to 22.58 per cent.

\section{Haulm}

Row spacing did not significantly influence the content and yield of all the quality parameters. Mainly due to the on par haulm dry matter yield recorded for row spacing, the non-significant yield of quality parameters were recorded.

\section{Seed quality}

Suggesting that seed quality is not affected by spacing, row spacing did not significantly influence the seed quality parameters namely germination percentage ( 87.5 to $88.0 \%$ ), field emergence, root length, shoot length, total seedling length $(33.0 \mathrm{~cm}$ to $33.86 \mathrm{~cm}$ ) and seedling vigour index (3109.6 to 3192.8 ).

\section{Effect of Seed Rate Growth and yield}

The yield, growth and yield components were not influence significantly by Seed rate. However, the higher seed yield $\left(739 \mathrm{~kg} \mathrm{ha}^{-1}\right)$ and haulm yield $\left(4118 \mathrm{~kg} \mathrm{ha}^{-1}\right)$ were recorded with $25 \mathrm{~kg} \mathrm{ha}^{-1}$ and $20 \mathrm{~kg} \mathrm{ha}^{-1}$ seed rate, respectively. Due to the on par growth and yield components, the on par yield recorded with seed rate.

\section{Proximate Composition (Quality) Seed}

These findings are in consistency with Santiesteban et al. (2002). The content and yield of seed quality parameters were not influenced significantly irrespective of the seed rates. On the other hand, the crude protein content ranged from 22.16 per cent to 22.87 per cent. Mainly due to the on par seed dry matter yield recorded for seed rates, non-significant yield of quality parameters were recorded.

\section{Haulm}

Mainly due to the on par haulm dry matter yield, seed rate did not influence significantly the content and yield of quality parameters in haulm.

\section{Seed quality}

Seed rate did not affect significantly the seed quality parameters viz., germination percentage ( 88.2 to $88.3 \%)$, field emergence, root length, and shoot length, total seedling length $(32.8 \mathrm{~cm}$ to $33.8 \mathrm{~cm})$ and seedling vigour index (3104.0 to 3185.2).

\section{Conclusion}

With $30 \mathrm{~cm}$ row spacing at $30 \mathrm{~kg} \mathrm{ha}^{-1}$ seed rate, fodder cowpea sown in June second fortnight results in significantly higher seed yield $\left(1054 \mathrm{~kg} \mathrm{ha}^{-1}\right)$ and haulm yield $\left(4968 \mathrm{~kg} \mathrm{ha}^{-1}\right)$.

\section{References}

[1]. ANGNe, M.N., PATIL, R.A., MAHADKAR, U.V. AND KHANVILKAR, S.A., 1993, Response of cowpea to nitrogen, phosphorus and stand geometry. Journal of Maharashtra Agricultural Universities, 18 (1): 121-122.

[2]. ARORA, N.D., MALIK, B.S. AND LODHI, G.P., 1971, Studies on the effect of row spacing and seed rate on the grain yield of cowpea (Vigna sinensis L.). Haryana Agricultural University Journal of Research, 3: 28.

[3]. DWIVEDI, D.K., SINGH, H., SINGH, K.M., SHAHI, B. AND RAI, J.N., 1994, Response of french bean (Phaseolus vulgaris) to population densities and nitrogen levels under mid-upland situation in North east alluvial plains of Bihar. Indian Journal of Agronomy, 39: 581 -583.

[4]. GOMEZ, K.A. AND GOMEZ, A.A., 1984, Statistical Procedures for Agricultural Research, An International Rice Research Institute Book, Wiley- Inter Science Publication, New York, USA, 680.

[5]. HARI AND GILL,S.S.,1981, Effect of seed size and sowing dates on seed crop of pea, Seed Research, 9 (2): 122-125. Mc EWEN, J., 1973, The effects of growth regulators, seed rates and row spacings on field beans (Vicia faba L.). Journal of Agricultural Sciences, Cambridge, 80:37-42.

[6]. Mc EWEN, J., 1973, The effects of growth regulators, seed rates and row spacings on field beans (Vicia faba L.). Journal of Agricultural Sciences, Cambridge, 80:37-42.

[7]. RAVINDER K. AND SINGH, N.P., 1998, Effect of date of sowing on growth and yield of cowpea [Vigna unguiculata (L.) walp]. Legume Research, 21 (1): 54-56

[8]. RUTKOWSKI, M. AND FORDONSKI, G., 1987, Effect of sowing and harvesting date on field bean yields. Biuletyn InstytutuHodowli -i- Aklimatyzacji- Roslin, 163: 165-174.

[9]. RAJESH, K. YADAV, B.D. AND JOON, R.K., 1998, The effect of inter and intra-row spacings and variety on the seed yield of cowpea. International Journal of Tropical Agriculture, 15 (1/4):233- 236.

[10]. SINGH, A.K., 2003, Role of National Dairy Development Board in forage and fodder seed production, Forage Research, 29 (1): 49-54 
[11]. SREelatha, D., RAO, K.L., VEeraRAghAVAiAH, R. AND PADMAJA, M., 1997, Physiological variations in french bean (Phaseolus vulgaris L.) cultivars as affected by sowing dates. Annals of Agricultural Research, 18: 111-114.

[12]. SANTIESTEBAN, S.R., ZOMORA, R.A., GOMEZ, P.E., VERDECIA, P.P., HERNANDEZ, G.L. AND ZAMORA, Z.W., 2002, Effect of sowing density on IITA Precoz [Vigna unguiculata (L.) Walp] in two seasons of the year. Alimentaria, 39 (332): 45-48.

[13]. SINGH, D.N. AND TRIPATHI, P., 1994, Effect of NPK fertilizers and spacing on growth and yield of french bean. Vegetable Science, 21 (1): 7-11.

[14]. YADAV, G.L., 2003, Effect of sowing time, row spacing and seed rate on yield of cowpea under rainfed condition. Indian Journal of Pulses Research, 16 (2): 157-158. 